\title{
The pathophysiology of chagasic megacolon: beyond ICC...
}

\author{
Gabrio Bassotti • Vincenzo Villanacci
}

Received: 31 October 2012 / Accepted: 8 November 2012 / Published online: 21 November 2012

(C) Springer-Verlag Berlin Heidelberg 2012

To the editor:

Dear Sir,

We read with interest the recent article by Adad et al. [1] in which they reported a reduction of the number of interstitial cells of Cajal (ICC) in the colonic myenteric plexus and circular muscle areas of chagasic patients with or without megacolon. This reduction was much more pronounced in patients with megacolon, where it was also accompanied by a significant decrease of enteric neurons [1]. The strength of this study relies in the fact that the authors took into consideration patients without overt megacolon and the cell count was carried out in complete intestinal rings that allowed them to correct the neuron and ICC dispersion due to intestinal dilation and muscular hypertrophy of the megacolon. The findings of ICC decrease were consistent with previous studies in chagasic patients with megacolon [2-4]. However, in our study, we found an increase of one of the three colonic populations of ICC, the intramuscular, and we disagree with the interpretation of Adad et al. that this increase might have been due to an inclusion of mast cells in the count, since these were specifically excluded, as specified in the paper [4]; on the other hand, in our experience the distribution of ICC within the human colon is fairly homogeneous (Bassotti and Villanacci, unpublished observations). More importantly, in their

\footnotetext{
G. Bassotti

Gastroenterology and Hepatology Section, Department of Clinical and Experimental Medicine, University of Perugia,

Perugia, Italy

\section{Villanacci}

Second Pathology Section, Spedali Civili,

Brescia, Italy

G. Bassotti $(\bowtie)$

Clinica di Gastroenterologia ed Epatologia, Ospedale Santa Maria della Misericordia, Piazzale Menghini, 1, 06156 San Sisto,

Perugia, Italy

e-mail: gabassot@tin.it
}

paper Adad et al. did not distinguish between the three well established populations of colonic ICC, i.e., ICC submucous (along the submucosal surface of the circular muscle bundle), ICC myenteric (within the intermuscular space between circular and longitudinal muscle layers, myenteric region, which displays the highest yield of ICC in normal tissue), and ICC intramuscular (within the muscle fibers of the circular and longitudinal muscle layers) [4]. This fact might have led to a decreased cell count due to a different methodologic approach.

However, we were pleased that Adad et al. did not limit their approach only to ICC, but included enteric neurons, that were also decreased. This fact, in addition to the significant decrease of enteric glial cells we also found in patients with megacolon and other conditions characterized by constipation $[4,5]$ strongly suggests that the development of megacolon requires enteric denervation and that an ICC decrease is an ancillary finding to neurogliopathic abnormalities.

\section{References}

1. Adad SJ, Silva GB, Jammal AA (2012) The significantly reduced number of interstitial cells of Cajal in chagasic megacolon (CM) patients might contribute to the pathophysiology of CM. Virchows Arch 461:385-392

2. Hagger R, Finlayson C, Kahu F, Oliveira R, Chimelli L, Kumar D (2000) A deficiency of interstitial cells of Cajal in chagasic megacolon. J Auton Nerv Syst 80:108-111

3. Geraldino RS, Ferreira AJ, Lima MA, Cabrine M, Ramirez LE (2006) Interstitial cells of Cajal in patients with chagasic megacolon originating from a region of old endemicity. Pathophysiology 13:71-74

4. Iantorno G, Bassotti G, Kogan Z, Lumi CM, Cabanne AM, Fisogni S, Varrica LM, Bilder CR, Munoz JP, Liserre B, Morelli A, Villanacci V (2007) The enteric nervous system in chagasic and idiopathic megacolon. Am J Surg Pathol 31:460-468

5. Bassotti G, Villanacci V (2011) Can "functional" constipation be considered as a form of enteric neuro-gliopathy? Glia 59:345-350 